\title{
COMUNICACIONES ORALES
}

\section{SÍNDROME DE ERASMUS EN EL ÁREA SANITARIA DE VIGO}

COMPLEXO HOSPITALARIO UNIVERSITARIO DE VIGO

Alonso Parada, María; Freire Dapena, Mayka; Álvarez Rodríguez, Elena; Vázquez Triñanes, Caritina; González González, Lucía; Villaverde Álvarez, Iria; Rivera Gallego, Alberto; Sopeña Pérez-Argüelles, Bernardo; Martínez Vázquez, César

INTRODUCCIÓN: La esclerosis sitémica (SSc) es una enfermedad sistémica autoimmune del tejido conectivo de etiología desconocida, aunque pueden jugar un papel factores genéticos y medioambientales. Desde la comunicación de la asociación de la exposición al sílice con la SSc por Erasmus en 1957, varios autores han corroborado su coexistencia, aunque con resultados muy dispares dependiendo de la población estudiada.

OBJECTIVOS: Describir las características clínicas y analíticas de pacientes con síndrome de Erasmus en nuestro área sanitaria.

Material y métodos: Revisión de las historias clínicas de los pacientes hospitalizados en el Complexo Hospitalario Universitario de Vigo desde 1994 a 2011 con el diagnóstico de SSc, seleccionando aquellos en los que se menciona exposición laboral al sílice.

RESULTADOS: Durante este tiempo el diagnóstico de SSc se confirmó en 86 pacientes. 10 enfermos (7 de los 13 hombres y 3 de las 73 mujeres) habían estado expuestos al sílice y fueron seleccionados para el estudio. 6 pacientes habían trabajado en canteras de granito, uno excavando túneles, y otros 3 en fábricas de cerámica. La media de edad al diagnóstico de esclerodermia fue de 51 años (rango 26-76). De acuerdo con la clasificación propuesta por LeRoy and Medsger seis eran variantes difusas y 3 limitadas. Otras dos fueron diagnosticadas de enfermedad mixta del tejido conectivo. El síntoma inicial fue fenómeno de Raynaud en el $50 \%$ y esclerosis cutánea en el $20 \%$, habiendo aparecido una media de 1 año antes del diagnóstico. Las manifestaciones clínicas al momento del diagnóstico fueron: disnea $90 \%$, RP $80 \%$, reflujo esofágico $70 \%$, úlceras digitales $40 \%$, telangiectasia $30 \%$, artritis $30 \%$, tendinopatía $20 \%$, calcinosis, arritmias complejas y crisis renal $10 \%$. La positividad para autoanticuerpos observada fue la siguiente: 10 ANA (1 patrón anticentrómero), 2 anti-dsDNA, 1 anti-SM, 1 anti-RNP, 6 anti-ScI70, 2 ACLA, 3 RF. En 9 pacientes se detectaron datos de enfermedad pulmonar intersticial en las pruebas de imagen. Se objetivó una disminución en el test de difusión en 7 de los 8 pacientes en los que se realizaron tests de función pulmonar, 5 de los cuales tenían un patrón restrictivo típico con capacidad vital forzada disminuída. Se realizó brocoscopia y biopsia transbronquial en 4 pacientes, con descripción en todas las muestras de fibrosis y depósitos de sílice. Se hizo ecocardiografía transtorácica en 7 pacientes, con una PASP estimada superior a $25 \mathrm{mmHg}$ in 5. Sólo fue realizado cateterismo cardíaco derecho en 2 enfermos, con medidas de confirmatorias de HTP en ambos. 7 pacientes fallecieron durante el seguimiento, una media de 4.2 años desde el diagnóstico. La causa de la muerte fue insuficiencia respiratoria en 3 casos, insuficiencia cardíaca refractaria en 2, cirrosis hepática en uno y desconocido en otro.

CONCLUSIONES: El síndrome de Erasmus es común entre los enfermos varones con SSc de nuestro área sanitaria que requieren ingreso hospitalario, con predominio de la variante difusa. El curso clínico de nuestros pacientes es rápidamente progresivo, con desarrollo frecuente de complicaciones mixtas cardiopulmonares y una corta supervivencia desde el diagnóstico.

\section{TRATAMIENTO DE ENFERMEDADES AUTOINMUNES HEMATOLOGICAS CON RITUXIMAB}

COMPLEJO HOSPITALARIO UNIVERSITARIO DE VIGO

González González, Lucía; Sopeña, Bernardo; Álvarez, Leticia; Rivera, Alberto; Freire Dapena, Maika; Vázquez Triñanes, Caritina; Argibay, Ana; Pérez Rodríguez, Ma Teresa; Martínez Vázquez, César

INTRODUCCION: El rituximab (RTX) es un anticuerpo monoclonal quimérico humanizado específico para el antígeno CD-20. El antígeno CD-20 es una diana adecuada para la inmunoterapia ya que su unión de anticuerpos antiCD-20 provoca una rápida depleción de los linfocitos $B$. RTX puede ser una opción terapéutica efectiva en pacientes con enfermedades hematológicas autoinmunes refractarias a otros tratamientos que podría evitar la esplenectomía.

OBJETIVOS: El objetivo de este estudio es establecer la eficacia del RTX en pacientes con anemia hemolítica autoinmune (AHA) y purpura trombocitopenia au- toinmune (ITP) refractarias a tratamientos convencionales y su tolerancia en una serie de pacientes con un seguimiento largo.

METODOS: Se incluyeron en este trabajo todos los pacientes que habían recibido tratamiento con RTX por citopenias autoinmunes refractarias a tratamientos convencionales en nuestro centro desde enero de 2004 hasta junio de 2011 con una edad superior a 16 años. Se recogieron las características clínicas, los resultados analíticos, los tratamientos previos y su evolución. Recibieron RTX (MABTHERA®, ROCHE) a dosis de $375 \mathrm{mg} / \mathrm{m}^{2}$ endovenosos excepto un paciente que recibió dosis de $1 \mathrm{~g}$.

RESULTADOS: Se analizaron 37 pacientes ( $54 \%$ mujeres) con una edad media de 63 años que habían fracasado a más de dos tratamientos previos, en 8 casos se había practicado la esplenectomia. ITP fue diagnosticada en 25 pacientes recibiendo RTX después de fracasos a tratamientos de primera elección presentando respuesta 16 de ellos (64\%) con un tiempo medio sin enfermedad de 23 meses, el 20\% de las ITP presentaba una enfermedad autoinmune asociada siendo en estos la respuesta al RTX del 100\%. RTX se administró a 11 pacientes con AHA refractaria con una respuesta completa al RT en 7 de ellos (64\%) con un tiempo medio libre de enfermedad de 25 meses, 4 pacientes además de AHA tenía otra patología autoinmune, evidenciándose respuesta al RTX en todos y mayor control de otras manifestaciones no hematológicas, dos de estos pacientes habían sido diagnosticados de hepatitis autoinmune con desaparición completa de clínica y parámetros de actividad relacionados con esta patología. Un solo paciente padecía simultáneamente ITP/AHA presentando repuesta completa y permanente desde hace 6 meses. La administración del tratamiento fue bien tolerado. Cuatro pacientes (10\%) desarrollaron durante el tiempo de seguimiento (media de 12 meses) neoplasias falleciendo tres de ellos. Otros cuatro pacientes presentaron infecciones no graves.

CONCLUSIONES: RTX es un tratamiento eficaz en pacientes con enfermedades hematológicas autoinmunes que fracasan a tratamientos de primera línea. La respuesta fue excelente en pacientes en el contexto de enfermedades autoinmunes. En el estudio se ha detectado un aumento en el desarrollo de neoplasias posttratamiento.

\section{CALIDAD DE LA BIOPSIA DE LA ARTERIA TEMPORAL EN ARTERITIS DE CÉLULAS GIGANTES EN EL ÁREA SANITARIA DE FERROL}

HOSPITAL ARQUITECTO MARCIDE

Ferreira González, Lucía; Caínzos Romero, Tamara; Trigás Ferrín, María; Vilariño Maneiro, Laura; Fernández Fernández, Francisco J.; Sesma, Pascual

INTRODUCCIÓN: A pesar de los avances en el diagnóstico y tratamiento de la arteritis de células gigantes, la biopsia de la arteria temporal continúa siendo el gold standard para el diagnóstico. Se recomienda un tamaño de biopsia no inferior a $2 \mathrm{~cm}$ para el diagnóstico óptimo, debido a la reducción que sufre el tejido durante la fijación.

OBJETIVO Y MÉTODOS: Revisar la calidad de las biopsias de la arteria temporal practicadas a una serie de pacientes con diagnóstico de arteritis de células gigantes durante el periodo Enero-2005 a Agosto-2011 en el Área Sanitaria de Ferrol. Los datos se recogieron de forma retrospectiva mediante el programa IANUS, identificando a los pacientes a través del servicio de codificación. Se incluyeron los casos que cumplían al menos 3 de los 5 criterios clasificatorios y se excluyeron aquellos pacientes en los que se había realizado un diagnóstico clínico sin obtención de biopsia. El análisis estadístico se realizó con el programa SPSS, versión 15.0

RESULTADOS: De un total de 32 biopsias realizadas, en el $53,1 \%$ de los casos se confirmó histopatológicamente el diagnóstico de sospecha de arteritis de células gigantes. En un caso la muestra remitida a Anatomía Patológica se descartó por corresponder a tejido venoso. El tamaño medio de las muestras fue de $0,7 \mathrm{~cm}$ $(0,2-2 \mathrm{~cm}$, DE 0,49). Sólo el 9,7\% (3 muestras) superaban el límite de 1,5 cm y en este caso el 66,7\% (2) de ellas fueron diagnósticas. En el restante 90,3\% de muestras, con tamaño inferior a $1,5 \mathrm{~cm}$, sólo el $50 \%$ resultó diagnóstica. No obstante, no se encontraron diferencias estadísticamente significativas en el tamaño de la muestra entre los pacientes con biopsia diagnóstica y los que no, con una media de 0,7 y $0,6 \mathrm{~cm}$ respectivamente $(p=0,6)$. En el $84,4 \%$ de los casos se indicó tratamiento con glucocorticoides antes de la realización de la biopsia, con una dosis inicial media de $53 \mathrm{mg}$ de prednisona (10-80 mg, DE 14,2). En 
el 6,3\% de los casos (2) se administraron bolos de metilprednisolona debido al debut con síntomas visuales. La duración del tratamiento con prednisona antes de la realización de la biopsia fue de 9,6 días de media (0-90 días, DE 16,6). № hubo diferencias estadísticamente significativas en cuanto a la rentabilidad de la biopsia entre los pacientes que habían recibido tratamiento esteroideo previo y los que no, aunque se apreció una media de duración del tratamiento en días inferior en los pacientes en los que la biopsia resultó diagnóstica (6,3 días frente a 13,1 días). No se encontraron diferencias en los valores de VSG entre los paciente con biopsia diagnóstica y aquellos con biopsia negativa, con una media de VSG en ambos grupos de 86 y $85 \mathrm{~mm}$ respectivamente $(p=0,9)$.

CONCLUSIÓN: La rentabilidad de las biopsias realizadas en nuestro centro ha sido baja, probablemente debido al reducido tamaño de las muestras y al tratamiento previo con glucocorticoides. En los pacientes con biopsia negativa, si bien no encontramos diferencias estadísticamente significativas, la duración del tratamiento con glucocorticoides había sido mayor. Debemos insistir tanto en la importancia de obtener un tamaño adecuado de muestra, al menos de $1,5 \mathrm{~cm}$, de cara a mejorar la sensibilidad de la biopsia como en la realización precoz de la misma una vez iniciado el tratamiento.

\section{ALTA TASA DE PRODUCTORES DE BETALACTAMASAS DE ESPECTRO AMPLIADO EN BACTERIEMIAS POR BACILOS GRAM NEGATIVOS}

\section{COMPLEXO HOSPITALARIO UNIVERSITARIO DE VIGO}

Pérez Rodríguez, María Teresa; Argibay, Ana; Longueira, Rebeca; Martínez Lamas, Lucía; Sousa, Adrián; Alonso, Ana; Rodríguez Gómez, Ana; Martínez Vidal, Ana; Martínez Vázquez, César

INTRODUCCIÓN-OBJETIVOS: Los bacilos Gram negativos (BGN) constituyen el segundo grupo de microorganismos aislados con mayor frecuencia en los hemocultivos. Los objetivos del estudio fueron conocer las características clínicas, terapéuticas y microbiológicas de los BGN (identificación, antibiograma) aislados en hemocultivos e identificar los factores asociados con la mortalidad.

PACIENTES Y MÉTODO: De forma prospectiva se recogieron las bacteriemias por BGN en pacientes mayores de 15 años entre enero y diciembre de 2011. Se revisaron las características epidemiológicas de los pacientes, el origen de la infección, el tratamiento empírico, así como la adecuación según el microorganismo aislado y la evolución posterior. Las bacteriemias se dividieron en adquiridas en la comunidad (BAC), nosocomiales (BN) 0 asociadas a cuidado sanitario (BCS).

RESULTADOS: De un total de 228 bacteriemias por BGN se seleccionaron aleatoriamente 100 , que se clasificaron en BAC $46 \%$, BN 35\% y BCS 19\%. El $54 \%$ eran varones con una edad media de $64 \pm 17.7$ años BN, $76 \pm 14.7$ años BAC, 68 \pm 11.8 años, $p=0.003$. El origen más frecuente de la bacteriemia fue el tracto urinario (43\%), seguido del abdominal (14\%), catéter (13\%), vía biliar (12\%) y pulmonar (12\%). La forma de presentación clínica fue fiebre $(68 \%)$, clínica abdominal (22\%), clínica urinaria (18\%) y shock séptico (11\%). El tratamiento empirico más frecuente fueron los betalactámicos $(52 \%, 33 \%$ BN, $70 \%$ BAC, $50 \% \mathrm{BCS}, \mathrm{p}=0.08)$, seguidos de meropenem ( $38 \%, 60 \% \mathrm{BN}, 16 \% \mathrm{BAC}, 44 \%$ $B C S, P=0.001)$ y quinolonas $(12 \%, 9 \% B N, 14 \% B A C, 13 \% p=0.84)$. Se utilizaron fármacos con actividad antipseudomónica en el $58 \%$ de los casos (76\% $\mathrm{BN}, 30 \% \mathrm{BAC}, 88 \% \mathrm{BCS} p=0.0001)$. El tratamiento fue inadecuado en el $15 \%$ de los casos (18\% BN, 16\% BAC, 6\% BCS, $p=0.53$ ). Los microorganismos aislados fueron $\mathrm{E}$. coli $(64 \%, 49 \% \mathrm{BN}, 83 \% \mathrm{BAC}, 47 \% \mathrm{BCS}, \mathrm{p}=0.002)$, $\mathrm{E}$. cloacae $(9 \%, 6 \% \mathrm{BN}, 4 \% \mathrm{BAC}, 26 \% \mathrm{BCS}, p=0.013)$, K. pneumoniae $(5 \%)$, S. marcescens $(5 \%)$, A. baumannii (3\%), P. mirabilis $(3 \%)$, B. stercoris $(2 \%)$. Sólo se obtuvo 1 aislamiento de los siguientes microorganismos: A. Iwoffi, B. cepacia, E. aerogenes, K. oxitoca, M. caterralis, P. oralis, P. stuartii, P. aeruginosa, P. fluorescens. El 19\% de los microorganismos eran productores de betalactamasas de espectro ampliado (BLEE) (26\% BN, 13\% BAC, $21 \%$ BCS, p $=0.34$ ), el $20 \%$ eran resistentes a Quinolonas (23\% BN, $17 \%$ BAC, $21 \%$ BCS, $p$ $=0.82$ ) y 3 aislamientos eran multirresistentes todos ellos BN. Dos aislamientos eran resistentes a carbapenémicos, en ambos casos el microorganismo aislado fue $A$. baumannii. Los factores de riesgo asociados con BLEE fueron: cirugía abdominal ( $O R=15.0$, IC 1.47-153.55), vía central $(O R=3.4$, IC 1.10-10.24), tratamiento antibiótico previo $(\mathrm{OR}=4.33$, IC 1.48-12.66) y tratamiento previo con Quinolonas $(\mathrm{OR}=5.13$, IC 1.15-22.83). Fallecieron 15 pacientes (17\% BN, $9 \% \mathrm{BAC}, 26 \% \mathrm{BCS}, p=0,18)$, los factores asociados fueron: insuficiencia renal (OR 3.5, IC 1.11-11.00) y shock séptico (OR 4.05, IC 1.02-16.13). Sin embargo, no se encontraron diferencias en cuanto al tratamiento inadecuado (17\% en los fallecidos y $15 \%$ en los no fallecidos, $p=1$ ).

CONCLUSIONES: Se ha detectado una elevada tasa de BLEE de bacteriemias por BGN. Los factores de riesgo asociados fueron cirugía abdominal, vía central y exposición previa a antibióticos (especialmente quinolonas).

\section{SEGUNDOS TUMORES PRIMARIOS EN PACIENTES VIH}

HOSPITAL XERAL-CIES, COMPLEJO HOSPITALARIO UNIVERSITARIO DE VIGO

Vaqueiro Rodríguez, Inés; Ocampo, Antonio; Miralles, Celia; Rodríguez da Silva, Alfredo;

Villaverde, Iria; Alonso Parada, María; Martínez Vázquez, César

INTRODUCCIÓN: Un segundo tumor primario (STP) representa la aparición de una neoplasia de diferente origen en personas con antecedentes de cáncer. Su prevalencia es del 5-16\%. La mayor supervivencia de los pacientes VIH conlleva un aumento de las enfermedades neoplásicas, sin embargo la información sobre STP en esta población es mínima

OBJETIVO: Analizar las características clínico-evolutivas de pacientes VIH con STP en nuestra área sanitaria.

MÉTODOS: Revisión retrospectiva del historial de los 2726 pacientes de la consulta de VIH del Hospital Xeral-Cíes desde 1989 hasta la actualidad. Se seleccionaron los casos con neoplasias malignas confirmadas y se analizaron las características de aquellos que presentaron un STP.

RESULTADOS: Desde 1989 se diagnosticaron 211 tumores en 200 pacientes. Los 11 pacientes con STP eran varones con diagnóstico de infección por VIH entre 1990 y 2002. La conducta de riesgo asociada fue el uso de drogas parenterales en 4 casos, homosexual en 5 y heterosexual en 2. La mediana del nadir de CD4 fue 54 (rango 5-396). Todos recibieron tratamiento antirretroviral (TARV) en algún momento de su evolución: $81,8 \%$ inhibidores de la proteasa, $54,5 \%$ ITINAN, 18,2\% raltegravir, 9,1\% enfuvirtida y $0 \%$ maraviroc. El recuento medio de linfocitos CD4 al diagnóstico de cáncer fue 381 (59-765) con el primer tumor primario (PTP) y 344 (7-1001) con el STP. Se observó una tendencia al descenso de la cifra de CD4 en el año previo al diagnóstico de ambos tumores. Tres y 8 pacientes recibían TARV al diagnóstico del PTP y STP respectivamente; en ambos casos, 3 pacientes tenían carga viral suprimida. La edad al diagnóstico del PTP fue 42,6 $\pm 10,67$ años, con una media de 7,9 años (0-17) de infección por VIH. E $44,4 \%$ de los PTP eran sarcomas de Kaposi (SK) y el resto eran neoplasias no definitorias de SIDA: 2 enfermedad de Hodgkin (EH), 2 carcinomas basocelulares, un carcinoma epidermoide de labio y un hepatocarcinoma (HCC). El 90,9\% de los pacientes recibió tratamiento específico para el PTP: 4 quimioterapia, 3 cirugía, 1 radioterapia y 3 otros tratamientos (interferon, radiofrecuencia y/o TARV). EI STP se diagnosticó entre 1 mes y 12 años tras el PTP (mediana 2 años) con una edad media de 45,4 $\pm 9,95$ años. Se diagnosticaron 3 linfomas no Hodgkin, 2 HCC, 2 tumores pulmonares, un SK, un cáncer de próstata, un seminoma y un carcinoma epidermoide de amígdala. No se halló relación entre las estirpes del PTP y del STP. Se trataron 9 casos de SPT: quimioterapia en 8, cirugía en 2 y radioterapia en uno. Fallecieron 8 pacientes $(72,7 \%)$ con una mediana de supervivencia de 4 meses (1-26). De todos los pacientes VIH con cáncer, el 12,5\% de los SK y el 9,5\% de los EH presentaron un STP.

CONCLUSIONES: En nuestra área, uno de cada 18 pacientes VIH con cáncer desarrolla un STP. Predominan en varones, con mal control virémico a pesar de TARV y tras un SK o una EH. No hay asociación clara entre la estirpe del PTP y del STP. Su pronóstico es desfavorable a corto plazo a pesar de recibir tratamiento específico.

\section{INFECCIÓN URINARIA DE ORIGEN COMUNITARIO POR E. COLI EN PACIENTES HOSPITALIZADOS}

HOSPITAL UNIVERSITARIO LUCUS AUGUSTI

Matesanz Fernández, María; Saavedra Martínez, Pablo; Iñiguez Vázquez, Iria; Rubal Bran, David; Monte Secades, Rafael; Rabuñal Rey, Ramón

INTRODUCCIÓN: La infección del tracto urinario (ITU) constituye la segunda infección de origen extrahospitalario atendida en hospitales, y figura entre las principales causas de ingreso y morbilidad en los servicios de Medicina Interna. Está presente en el $15-20 \%$ de las mujeres mayores de 80 años, el 30-40\% en ancianas hospitalizadas 0 ingresadas en instituciones geriátricas y prácticamente el $100 \%$ de portadores de sonda vesical permanente. En el varón, aumenta a partir de los 50 años en relación a la patología prostática y/o manipulaciones urológicas. OBJETIVO: Describir las características de la ITU adquiridas en la comunidad que precisan ingreso, y su relación con la dependencia, comorbilidad e institucionalización.

PACIENTES Y MÉTODO: estudio prospectivo de todos los pacientes adultos ingresados en el HULA entre el 23 de Enero de 2012 y el 24 de Febrero de 2012 con ITU adquirida en la comunidad. Se recogieron edad, sexo, servicio de ingreso, tipo de microbio, antibiograma, portador de sonda vesical, y se calcularon los índices de Barthel y Charlson. Para el análisis se utilizaron técnicas habituales de la estadística descriptiva mediante el paquete estadístico SPSS.

RESULTADOS: Se recogieron 52 pacientes, edad media 80,4 años, el 64,9\% mujeres. El microbio aislado con más frecuencia fue E. Coli (57,9\%); el 8,77\% de 
las infecciones fueron polimicrobianas. De los pacientes con infección por E. Coli (edad media 83,6 años, 78,8\% mujeres), el 53,3\% presentaba dependencia al menos moderada (Barthel < 60 ), el $9 \%$ estaba institucionalizado y el $9,1 \%$ portaba sonda permanente; el 15,2\% habían presentado ITU de repetición y el 27,3\% habían recibido antibióticos en los dos meses previos.

Los pacientes con dependencia y/o comorbilidad presentaron con más frecuencia resistencias a los antibióticos testados, aunque estas no fueron significativas.

Los pacientes que habían recibido antibióticos en los dos meses previos (el 100\% de los pacientes institucionalizados, el $22 \%$ de los no institucionalizados y el $66,7 \%$ de los portadores de sonda permanente) presentaron con más frecuencia resistencia a amoxicilina clavulánico $(66,7 \% p=0.04$, siendo la mayoría de estos sensibles a gentamicina) y ciprofloxacino $(53,3 \% p=0,05)$, no encontrándose resistencias significativas para ceftriaxona.

CONCLUSIÓN: El microbio más frecuente aislado en infecciones urinarias en nuestro medio es el E. Coli. La toma de antibióticos en los dos meses previos se asocia a resistencia para amoxicilina-clavulánico y ciprofloxacino.

En nuestro medio, ante sospecha de ITU y tratamiento antibiótico previo o institucionalización, es recomendable iniciar tratamiento empírico con ceftriaxona o con una asociación de amoxicilina clavulánico más aminoglucósido.

\section{RELACIÓN ENTRE TROMBOSIS VENOSA RESIDUAL Y CIFRAS DE D DÍMEROS AL DIAGNÓSTICO Y SEGUIMIENTO TRAS UN EPISODIO DE TROMBOSIS VENOSA PROFUNDA}

MEDICINA INTERNA. HOSPITAL ARQUITECTO MARCIDE

Barbagelata López, Cristina; Mella Pérez, Carmen; Fernández Bouza, Elena; Vázquez Vázquez, Begoña; Cainzos Romero, Tamara; Sardina Ferreiro, Raquel; Gómez Buela, Inmaculada; Rivas Costa, Gonzalo; Sesma Sánchez, Pascual

INTRODUCCIÓN: En el seguimiento de pacientes tras una trombosis venosa profunda (TVP), se ha descrito la presencia de trombosis residual (TR) en ecodoppler de control y cifras elevadas de D Dímero (DD) en el seguimiento, con un mayor riesgo de trombosis tras suspensión de la anticoagulación.

OBJETIVOS: Establecer si existe correlación en el seguimiento tras TVP entre la presencia de TR y cifras de DD elevados. Evaluar si existe algún factor clínico relacionado con la presencia de TR o DD elevados.

MATERIAL Y MÉTODOS: Estudio observacional, prospectivo, de los pacientes con diagnóstico de primer episodio de TVP entre enero del 2009 y junio del 2011, realizándose a los seis meses ecodoppler de control en la extremidad afecta y DD antes de suspender anticoagulación. Se realizó un análisis descriptivo de las variables incluidas en el estudio. Las variables cualitativas se expresan como frecuencias absolutas y porcentajes y las variables cuantitativas como medias y desviaciones estándares, para el análisis de las variables cuantitativas se utilizó el test de la t de Student y para las cualitativas el test de chi cuadrado utilizando paquete software SPSS 11.0.

RESULTADOS: Los 44 casos analizados presentaban una edad media de 62 años, $59 \%$ mujeres. El valor medio de DD al diagnóstico fue de $9135 \mathrm{ngr} / \mathrm{ml}$ (rango 440->20.000). La TVP fue provocada en el $61.4 \%$ de los casos, $13.7 \%$ antecedente de fractura o cirugía traumatológica en el mes previo, 38'6\% inmovilización mayor de 3 días, 9'1\% toma de anticonceptivos orales, 6’8\% neoplasia activa, 2 '3\% síndrome antifosfolípido. Otros factores de riesgo fueron: varices $366^{\prime} 4 \%$, obesidad $47^{\prime} 7 \%$, movilidad basal limitada $41 \%$. Las características del evento tvp mostraron: oclusión total $79 \%$, varios territorios $66 \%$ (iliaco-femoral $18 \%$, fémoro-poplíteo $47^{\prime} 7 \%$ ) y un solo territorio 34\% (femoral 12\%, poplíteo $15^{\prime} 9 \%$ y cayado de la safena 6'8\%). Se detectó TR a los 6 meses en el $43^{\prime} 2 \%$. Cifra media de de DD en el seguimiento $348 \mathrm{ngr} / \mathrm{ml}$ (rango 31-850). La presencia de fractura 0 cirugía traumatológica en el mes previo fue claramente más frecuente en el grupo de pacientes con TR (83'3\% frente a 16'7\%) aunque no alcanzó la significación estadística, la tendencia fue clara $(p=0.045)$. Respecto al análisis de otras variables no encontramos relación entre la presencia de TR con varices, obesidad, TVP provocada, movilidad basal limitada o inmovilización ni cáncer. En cuanto al tipo de evento, no encontramos relación entre TR y TVP de varios segmentos frente a un territorio, ni oclusión total frente a parcial. Tampoco encontramos relación con el valor de DD más elevado al diagnóstico y TR para valores de DD > de 5000 $0>10.000$,ni relación entre cifras de DD en seguimiento $>1500>250$ con la presencia de TR.

CONCLUSIONES: El 43’2\% de los pacientes con primer episodio de TVP atendidos en nuestra consulta presentan TR a los 6 meses de tratamiento anticoagulante. No encontramos asociación entre cifras elevadas de DD a los 6 meses y la presencia de TR, sin embargo claramente la presencia de TR es más frecuente en pacientes con fractura o cirugía traumatológica en el mes previo, en contra de lo publicado en otras series. No se encontró relación entre la presencia de trombosis residual y evento de TVP iliaco-femoral frente a fémoro-poplíteo u oclusión total frente a parcial.

\section{FILTROS DE VENA CAVA INFERIOR RECUPERABLES}

MEDICINA INTERNA. H. XERAL DE VIGO'. RADIOLOGÍA INTERVENCIONISTA. H. XERAL DE VIGO2. MEDICINA INTERNA. UNIDAD DE TROMBOSIS Y VASCULITIS. H. XERAL DE VIGO3

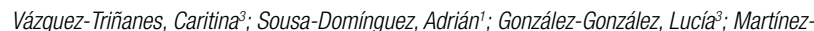
Vidal, Ana Isabel'; Rodríguez-Gómez, Ana Maria'; Casal, Moisés²; Freire Dapena, Mayka3; Rivera Gallego, Alberto'; Sopeña Pérez-Argüelles, Bernardo

INTRODUCCIÓN: Existen 3 tipos de filtros de vena cava (FVC): permanentes, temporales y recuperables. Actualmente se está imponiendo el uso de filtros recuperables que ofrecen la posibilidad de retirarse a corto-medio plazo o de mantenerse de forma permanente. A pesar de los resultados positivos en recientes estudios con este tipo de filtros, hay diversas cuestiones que siguen sin resolver: la adecuación de las indicaciones, el tiempo máximo apropiado de implantación y la posibilidad de retirada eficaz y segura.

OBJETIVOS: Valorar la adecuación de las indicaciones de los FVC y la eficacia y seguridad de los mismos en nuestro medio.

MATERIAL Y MÉTODOS: Se seleccionaron de la base de datos prospectiva de servicio de Radiología Intervencionista del CHUVI los pacientes sometidos a implante de FVC desde el 1/enero al 31/diciembre de 2011. Se consideraron complicaciones del FVC la muerte derivada de su implantación o retirada, la hemorragia, trombosis o infección del punto de acceso cutáneo, la formación de pseudoaneurisma, la migración del filtro, la perforación o trombosis de la vena cava inferior y el síndrome postrombótico. Se obtuvieron de las historias clínicas los datos demográficos, clínicos, analíticos y evolutivos para su análisis con el programa estadístico SPSS 18.0. Se utilizaron las medidas estadísticas: media $\pm D S$, rango y porcentaje.

RESULTADOS: Fueron sometidos a implante de FVC, todos ellos recuperables, 15 pacientes. La edad media fue de 66,8 16,34 años (rango $21-95$ ) y un $53 \%$ eran mujeres. Las indicaciones más frecuentes fueron el puente a la anticoagulación en cirugía programada o en otras situaciones de riesgo hemorrágico $(53 \%)$ y la hemorragia grave (33\%). Otras indicaciones fueron 1 recurrencia de TEP a pesar de dosis óptima anticoagulante de HBPM y 1 trombo flotante en vena ilíaca durante un brote de enfermedad de Crohn. La recurrencia de TEP durante el tiempo de permanencia del FVC ocurrió en 1 paciente (7\%), a los 64 días de la implantación, cuya indicación había sido ETV recurrente a pesar de dosis óptima de anticoagulación. La paciente padecía una neuropatía periférica no filiada, progresiva, y falleció a los 2 días de este nuevo TEP en insuficiencia respiratoria por hipoventilación. De los 13 pacientes con indicación por puente a la anticoagulación o por hemorragia $1(8 \%)$ tuvo una trombosis venosa profunda en miembro inferior mientras estuvo sin anticoagulación. No hubo ninguna complicación derivada del implante de los FVC. El FVC se retiró en 8 pacientes (53\%), sin ninguna complicación, de media a los 24,8 días de la implantación (rango 14-46). Todos estaban con dosis óptima de anticoagulación. En 7 pacientes (47\%) se decidió, debido a corta esperanza de vida, mantener el FVC de forma permanente. 5 de ellos fallecieron (71\%), 4 por progresión de cáncer y 1 por recurrencia de TEP y en hipoventilación por neuropatía periférica. Ninguno de los 7 presentó complicaciones derivadas del FVC durante

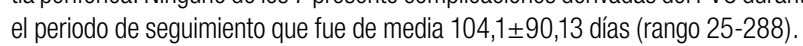
CONCLUSIONES: Las indicaciones más frecuentes de FVC en nuestro medio fueron el puente a la anticoagulación y la hemorragia, adecuándose a las recomendaciones actuales. El FVC en estas situaciones es eficaz en la prevención de TEP y es seguro.

\section{FACTORES DE RIESGO ASOCIADOS CON BACTERIEMIA POR S. EPIDERMIDIS RESISTENTE A LINEZLID}

UNIDAD DE PATOLOGÍA INFECCIOSA. MEDICINA INTERNA. COMPLEXO HOSP. UNIV. DE VIGO'; SERVICIO DE MICROBIOLOGÍA. COMPLEXO HOSP. UNIV. DE VIGO²

Pérez Rodríquez, María Teresa'; Lorenzo Castro, Rut'; Martínez Lamas, Lucía'; Argibay, Ana ${ }^{7}$, Sousa, Adrián ${ }^{1}$; Nodar, Andrés ; Álvarez Fernández, Maximiliano²; Martínez Vázquez, César

INTRODUCCIÓN/OBJETIVOS: Staphylococcus epidermidis es uno de los principales microorganismo aislados en hemocultivos, pudiendo ocasionar infecciones graves como sepsis por catéter, endocarditis, infección de dispositivos endovasculares... El tratamiento suele realizarse con vancomicina, linezolid 0 daptomicina, ya que más del $80 \%$ de las cepas son resistentes a oxacilina. Durante los últimos años se ha observado la aparición de clones de S. epidermidis resistentes a linezolid (SeRL), en muchos casos asociados al tratamiento previo con este fármaco. Los objetivos del trabajo fueron conocer la prevalencia de SeRL aislados en sangre y establecer los factores asociados con la aparición de la resistencia. 
MATERIAL Y MÉTODO: Se realizó un estudio de casos y controles. Se identificaron los aislamientos de SeRL en hemocultivos entre noviembre de 2009 y noviembre de 2011 y se seleccionó aleatoriamente un número igual de bacteriemias por S. epidermidis sensibles a linezolid (SeSL). Se recogieron los antecedentes médicos de los pacientes, tiempo de estancia hospitalaria hasta la bacteriemia, la exposición previa a Linezolid, estancia previa en Unidad de Críticos (UC). Los aislamientos se clasificaron en: contaminantes, asociados a infección de catéter, endocarditis, infección de dispositivo endovascular (válvula cardíaca, marcapasos, prótesis vascular). Se consideraron aislados resistentes los que presentaron una $\mathrm{CMl}>8 \mu \mathrm{g} / \mathrm{dl}$ por el método de E-test $®$ (bioMérieux, France).

RESULTADOS: De un total de 652 aislamientos de $\mathrm{S}$. epidermidis en hemocultivos se identificaron 53 SeRL (8\%), pertenecientes a 37 pacientes. Las características epidemiológicas de los paciente con bacteriemia por SeRL y SeSL fueron similares. La edad media fue de $66 \pm 14.2$ años y 45 eran varones (61\%). Las bacteriemias se clasificaron como bacteriemia recurrente (3\% de los SeRL y el $6 \%$ de los SeSL, $p=0.60$ ), infección de catéter ( $49 \%$ de SeRL y $62 \%$ de SeSL, $p=$ 0.34), contaminante ( $46 \%$ de SeRL y $29 \%$ de SeSL, $p=0.22$ ). Hubo 1 paciente con SeRL con endocarditis sobre válvula nativa y no se encontró ningún caso de infección de dispositivo endovascular. Los factores asociados con el aislamiento en hemocultivos de SeRL fueron: ingreso en REA (OR 10.7, IC = 2.80-41.21), tratamiento previo con Linezolid (OR 32.31, IC 6.68-156.34), ser portador de una vía central en el momento de la bacteriemia (OR 5.74, IC 2.09-15.77) o en la semana previa (OR 7.35, IC 2.63-20.60) y un mayor tiempo de ingreso (34 \pm 20.8 frente $15 \pm 16.3, p=0.0001)$. Como factor protector se encontró el ingreso en planta médica (OR 0,14, IC 0.04-0.48). La neoplasia hematológica fue menos frecuente en el grupo de SeRL (3\% frente a 19\%) aunque no se pudo demostrar diferencia $(p=0.056)$. En cuanto a la evolución, se observó una mayor tasa de recurrencia de la bacteriemia en SeRL ( $32 \%$ frente $5 \%, p=0.006$ ) y menor tasa de curación (32\% frente $78 \%, p=0.0001$ ) y no se demostró diferencia en cuanto a la mortalidad entre ambos grupos ( $35 \%$ frente $16 \%, p=0.11$ ). Los aislamientos de SeRL fueron con mayor frecuencia resistentes a oxacilina (100\% frente $73 \%, p=$ $0.001)$. Se identificaron 4 aislamientos con sensibilidad intermedia a glicopépticos (2 SeRL y 2 SeRL) y uno resistente a teicoplanina (SeRL).

CONCLUSIONES: La tasa de aislamientos de SeRL en nuestro centro es elevada (8\%), siendo clínicamente relevante en más del $50 \%$ de los pacientes. Los factores asociados con el aislamiento de SeRL fueron estancia hospitalaria prolongada, ingreso en REA, tratamiento previo con linezolid y ser portador de vía central en el momento de la bacteriemia o en la semana previa.

\section{MANEJO CONSERVADOR DE LA INFECCIÓN DE PRÓTESIS VASCULAR. ESTUDIO PROSPECTIVO DE 21 CASOS}

UNIDAD DE PATOLOGÍA INFECCIOSA. MEDICINA INTERNA. COMPLEJO HOSPITALARIO UNIVERSITARIO DE VIGO

Martínez Vázquez, César; Nodar Germiñas, Andrés; Pérez Rodríguez, María Teresa; Argibay Filgueira, Ana Belén; Portela, D.; Sopeña Argüelles, Bernardo; Vázquez Triñanes, Matilde Caritina; González González, Lucía; Villaverde, I.; Alonso, M.; Martínez Vidal, Ana Isabel; Rodríguez Gómez, Ana María; Sousa Domínguez, Adrián; Lorenzo Castro, Rut

INTRODUCCIÓN: En estos últimos años varias publicaciones han evidenciado la posibilidad de curación de un porcentaje elevado de prótesis vasculares infectadas, sin retirada de la misma, siempre y cuando se reúnan unas condiciones y a base de drenaje local y antibioterapia prolongada. Presentamos a continuación la experiencia en nuestro Centro con el manejo conservador de esta grave complicación.

PACIENTES Y MÉTODOS: Se incluyen de manera prospectiva observacional a todos los pacientes diagnosticados de infección vascular protésica en base a test microbiológicos, pruebas de imagen y clínica compatible, diagnosticados desde 2005 a 2009. Para ser incluidos la prótesis debe seguir permeable, la anastomosis sin fugas y estar hemodinámicamente estables.

Los pacientes son sometidos, cuando hay abscesos periprotésicos de un diámetro superior a $3 \mathrm{~cm}$, a drenaje quirúrgico 0 drenaje mediante Radiología Intervencionista y a tratamiento antibiótico parenteral de inducción durante 4 semanas, seguido de tratamiento antibiótico de consolidación, adecuados al germen, hasta tener evidencia de curación en base a la normalización de pruebas de imagen, radioisótopos, reactantes de fase y curación clínica. Una vez retirado el antibiótico se hace un seguimiento clínico-analítico de los pacientes durante 2 años para considerarlos curados.

RESULTADOS: Se reclutan 21 pacientes de los cuales no hubo seguimiento en 2 , de los 19 restantes 4 fueron considerados como fracaso terapéutico (3 en la fase de inducción antibiótica parenteral y 1 en el seguimiento tras retirada del antibióti-

co). Los 3 pacientes considerados fracaso terapéutico fueron sometidos a retirada de prótesis evolucionando favorablemente y el cuarto paciente curó aplicando de nuevo el protocolo de tratamiento conservador. En los 15 restantes (78\%) el manejo conservador fue exitoso.

CONCLUSIONES: Determinados enfermos con infección protésica vascular pueden ser curados, conservando su prótesis, con manejo conservador.

At volecea quidellaut lamus ma con nobite voloreptae porrum id modipsam et aut pra nihit estiaspit poriandus.

Uga. Rionsequi accupti nonsequiae dunt eiur suntio et molo beaqui doluptam ea vent aborereicia nienihi llecume sime et as comnis reptas re numquae cereptas 